\title{
Uterine Corpus Cancer pTis TNM Finding v6
}

National Cancer Institute

\section{Source}

National Cancer Institute. Uterine Corpus Cancer pT is TNM Finding v6. NCI Thesaurus.

Code C61341.

Uterine corpus cancer in situ. (from AJCC 6th Ed.) 